\title{
Sutureless Aortic Valve Replacement in a Patient with Transfemoral Aortic Valve Replacement and Left Ventricular Hypertrophy
}

\author{
Torsten Christ ${ }^{1} \quad$ Pascal M. Dohmen $^{1} \quad$ Michael Laule $^{2} \quad$ Karl Stangl $^{2} \quad$ Wolfgang Konertz $^{1}$ \\ ${ }^{1}$ Department of Cardiovascular Surgery, Charité-Universitätsmedizin \\ Berlin, Berlin, Germany \\ 2 Department of Cardiology and Angiology, Charité- \\ Universitätsmedizin Berlin, Berlin, Germany \\ Address for correspondence Torsten Christ, MD, Department of \\ Cardiovascular Surgery, Charité-Universitätsmedizin Berlin, \\ Charitéplatz 1, Berlin 10117, Germany \\ (e-mail: torsten.christ@charite.de).
}

Thorac Cardiovasc Surg Rep 2016;5:21-23.

\begin{abstract}
Keywords

- aortic valve

- heart valve

- TAVI

- cardiomyopathy

- sutureless aortic valve

Background Transarterial valve intervention (TAVI) is valuable in high-risk patients, however, in case of left ventricular outflow tract (LVOT) obstruction, conventional surgery, including partial myectomy, is indicated.

Case Description An 84-year-old female patient presented with increasing fatigue after TAVI in 2012, demonstrated a narrowed LVOT. Conventional surgery was performed, including removal of the transcathether valve, partial septal myectomy, and implantation of a sutureless valve. The postoperative course was uncomplicated. Conclusion Aortic valve stenosis combined with severe left-ventricular hypertrophy is not ideal for TAVI. Conventional surgery, performing partial septal myectomy and implantation of sutureless aortic prosthesis, seems more appropriate.
\end{abstract}

\section{Background}

Transaortic valve intervention (TAVI) is a valuable technique in inoperable and very high-risk patients with severe aortic valve stenosis. ${ }^{1}$ These patients often show secondary left ventricular hypertrophy due to chronic pressure overload. In case of left ventricular outflow tract (LVOT) obstruction, partial septal myectomy should be considered the most appropriate treatment for most patients. ${ }^{2}$

\section{Case Description}

An 84-year-old female patient presented with increasing fatigue after transfemoral valve implantation 2 years ago at another hospital. A 23-mm Edwards sapien (Edwards Lifesciences LLC, Irvine, CA) transcatheter heart valve was implanted. Echocardiographic examination demonstrated severe narrowing of the LVOT at $12 \mathrm{~mm}$ with a peak pressure gradient of $30.0 \mathrm{~mm} \mathrm{Hg}$ due to severe septal hypertrophy (-Fig. 1) and an elevated pressure gradient

received

March 21, 2015

accepted

April 16, 2015

published online

July 10, 2015
DOI http://dx.doi.org/ 10.1055/s-0035-1554991. ISSN 2194-7635. across the valve. Invasive pressure measurement showed a peak pressure gradient of $74.0 \mathrm{~mm} \mathrm{Hg}$ between the left ventricle and the ascending aorta. After clarifying therapeutic options for the patient (EuroSCORE: 10.1\%, EuroSCORE II: 7.54\%, STS score: $16.9 \%$, conventional surgery, including sternotomy, aortic cross-clamp, and cardiopulmonary bypass was preferred. The Edwards sapien aortic valve was explanted and the calcified native cusps were removed. The prosthetic valve showed thick pannus formation toward the LVOT ( $\mathbf{F i g}$. 2A), which was confirmed histologically by a pentachrome staining ( - Fig. 2B). Additionally, thrombotic material was found at the cusps toward the aorta. Partial septal myectomy was performed and a sutureless aortic valve (Sorin Perceval Size M [Sorin Group, Milan, Italy]) was implanted. Cross-clamp and cardiopulmonary bypass time was 25 and 43 minutes, respectively. The postoperative course of the patient was uncomplicated with stable sinus rhythm and no atrioventricular block occurred. Echocardiography showed an enlarged LVOT (-Fig. 3) with a peak pressure gradient of $13 \mathrm{~mm} \mathrm{Hg}$, a
License terms

Stuttgart · New York

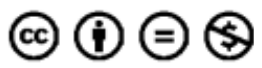




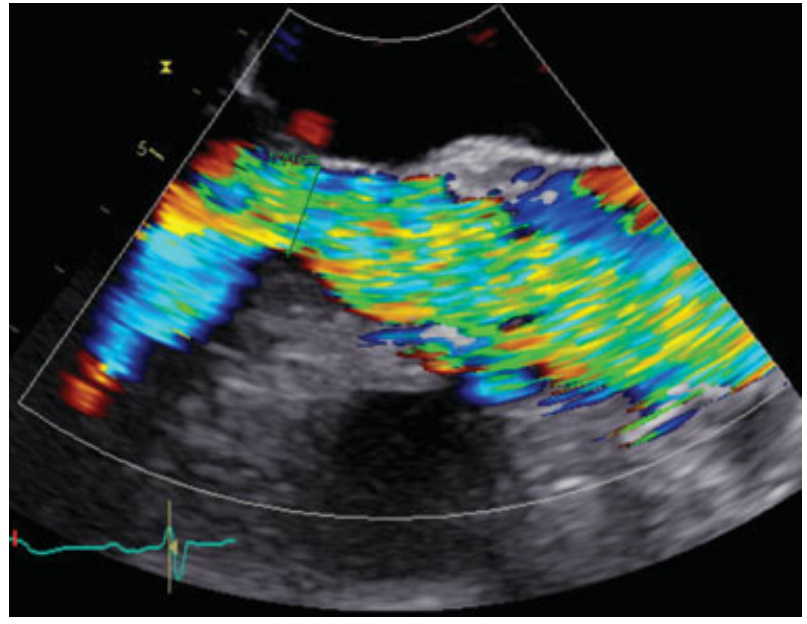

Fig. 1 Obstruction of the left ventricular outflow tract in color echocardiography before conventional surgery.

normal function of the prosthesis, and no ventricular septal defect.

\section{Conclusion}

High-risk patients suffering from severe aortic valve stenosis in combination with extensive left ventricular hypertrophy may not be the ideal candidates for TAVI. Even though hemodynamic results after TAVI were reported excellent, ${ }^{3}$ no resection of the obstruction in the LVOT is possible. Therefore, pressure overload after the TAVI-procedure persists and regression of left ventricular mass is either slowed down or inhibited. Even minor obstructions in the LVOT can be responsible for this process. This led in the reported case even to an increase of left ventricular obstruction, which had 2 years ago not been considered to be relevant. The LVOTobstruction caused consecutively hemodynamic turbulences, which are known to trigger the growth of pannus. ${ }^{4}$ This led to

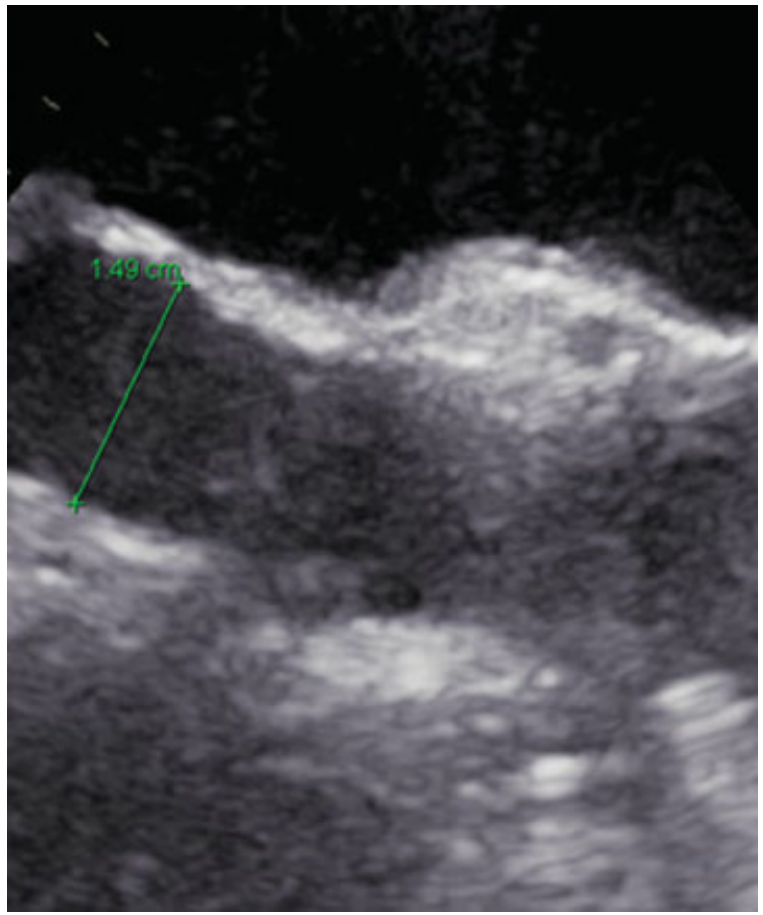

Fig. 3 Enlarged left ventricular outflow tract in echocardiography after conventional surgery including septal myectomy.

an even worse obstruction and the development of an vicious circle of LVOT-narrowing. Finally, the necessity of surgical valve replacement in combination with a septal myectomy arose. This procedure should be considered even in patients with no evident LVOT-obstruction, but extensive left ventricular hypertrophy.

Sutureless valve replacements or fast deployment aortic valves offer advantages in these high-risk patients. With simplified implantation techniques, the operation can be performed with minimized myocardial ischemic time $e^{5,6}$ and therefore reduced procedural risk. ${ }^{7}$ Furthermore,

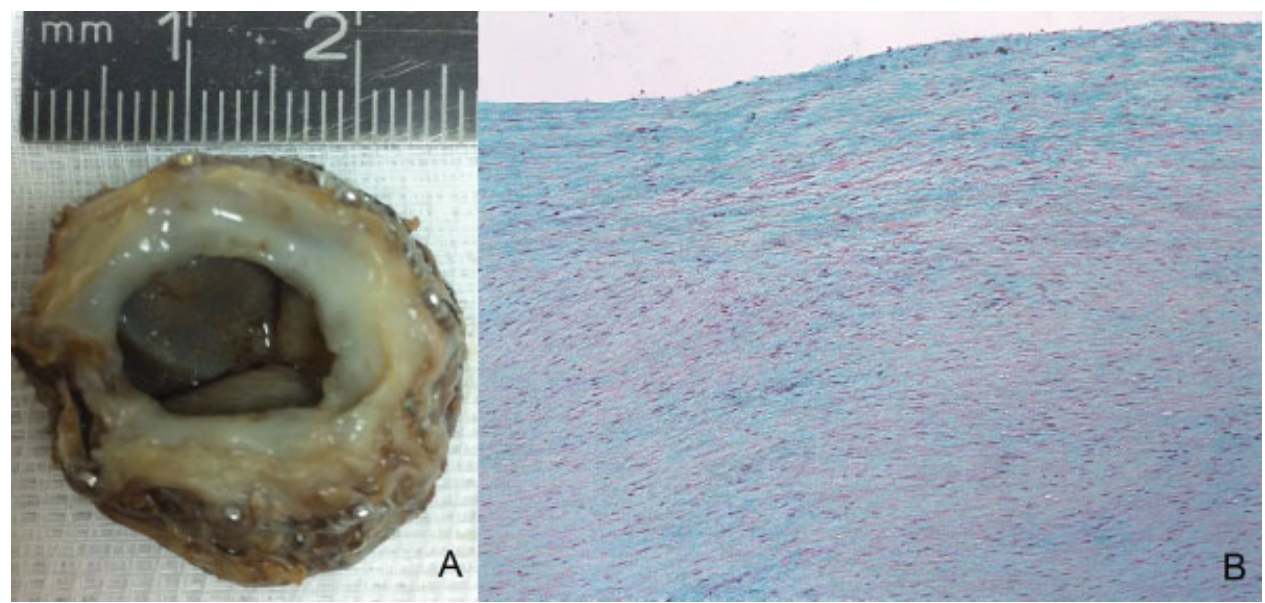

Fig. 2 (A) Explanted heart valve with thick pannus formation toward the left ventricular outflow tract. (B) Pentachrome staining of pannus formation. 
regression of left ventricular mass benefits from the septal myectomy, to prevent the vicious circle of LVOT obstruction.

Therefore, we conclude that conventional surgery performing partial septal myectomy and surgical valve replacement seems to be the appropriate option for high-risk patients with extensive left ventricular hypertrophy and even slight LVOT obstruction.

\section{References}

1 Svensson LG, Blackstone EH, Rajeswaran J, et al; PARTNER Trial Investigators. Comprehensive analysis of mortality among patients undergoing TAVR: results of the PARTNER trial. J Am Coll Cardiol 2014;64(2):158-168

2 Gersh BJ, Maron BJ, Bonow RO, et al; American College of Cardiology Foundation/American Heart Association Task Force on Practice; American Association for Thoracic Surgery; American Society of Echocardiography; American Society of Nuclear Cardiology; Heart Failure Society of America; Heart Rhythm Society; Society for Cardiovascular Angiography and Interventions; Society of Thoracic Surgeons. 2011 ACCF/AHA guideline for the diagnosis and treatment of hypertrophic cardiomyopathy: a report of the
American College of Cardiology Foundation/American Heart Association Task Force on Practice Guidelines. J Thorac Cardiovasc Surg 2011;142(6):e153-e203

3 Spethmann S, Dreger H, Baldenhofer G, et al. Long-term Doppler hemodynamics and effective orifice areas of Edwards SAPIEN and medtronic CoreValve prostheses after TAVI. Echocardiography 2014;31(3):302-310

4 Sakamoto Y, Hashimoto K, Okuyama H, Ishii S, Shingo T, Kagawa H. Prevalence of pannus formation after aortic valve replacement: clinical aspects and surgical management. J Artif Organs 2006; 9(3):199-202

5 Borger MA, Moustafine V, Conradi L, et al. A randomized multicenter trial of minimally invasive rapid deployment versus conventional full sternotomy aortic valve replacement. Ann Thorac Surg 2015;99(1):17-25

6 Rubino AS, Santarpino G, De Praetere H, et al. Early and intermediate outcome after aortic valve replacement with a sutureless bioprosthesis: Results of a multicenter study. J Thorac Cardiovasc Surg 2014;148(3):865-871, discussion 871

7 Borger MA, Dohmen P, Misfeld M, Mohr FW. Current trends in aortic valve replacement: development of the rapid deployment EDWARDS INTUITY valve system. Expert Rev Med Devices 2013; 10(4):461-470 